Ann. Zootech., I97I, 20 (4), 465-475.

\title{
UTILISATION COMPARÉE DU BLÉ, DE L'ORGE ET DU MAÏS DANS LA RATION DES AGNEAUX A L'ENGRAIS
}

\author{
D. AGUER, M. THÉRIEZ et G. MOLÉNAT \\ Station de Recherches sur l'Élevage des Ruminants, \\ Centre de Recherches de Clermont-Ferrand, I. N. R. A., \\ 63 - Saint-Genès-Champanelle
}

\begin{abstract}
RÉSUMÉ
Nous avons comparé l'utilisation de différentes rations riches en céréales (orge, blé ou maïs) par des agneaux mâles entre 20 et $32 \mathrm{~kg}$. Les rations étaient constituées d'aliment granulé contenant 73 p. Ioo de céréales et de foin normal, tous deux distribués ad libitum.

Les agneaux ont consommé des quantités voisines de matière sèche totale, mais ceux qui recevaient l'aliment maïs ont consommé moins de foin ( 8 p. Ioo de la matière sèche de la ration totale au lieu de 22 et 25 p. IOO).

L'évolution des quantités ingérées a fait apparaître un faible niveau d'ingestion de l'aliment concentré à base de blé tout au long de la période considérée. L'aliment " maïs " a, par contre, toujours été consommé en quantités élevées. Dans le cas de l'orge les quantités ingérées sont restées intermédiaires entre celles des deux autres aliments. Dans tous les cas, les consommations ont augmenté jusqu'au poids vif de $30 \mathrm{~kg}$ environ, puis se sont stabilisées au niveau de go $\mathrm{g}$ de matière sèche par kg de poids métabolique.

Quelle que soit la céréale utilisée, les agneaux ont eu des gains de poids vif élevés : 302 à $340 \mathrm{~g} / \mathrm{j}$, sans pour autant présenter d'accidents digestifs.

Les agneaux ingérant l'aliment " maïs " ont eu des rendements de carcasse significativement plus élevés que ceux des autres lots. Ils ont ingéré I 5 p. Ioo d'énergie (UF) de plus que ceux qui recevaient l'orge ou le blé, et ont eu des gains de poids vif et de carcasse supérieurs respectivement de I 2 et 28 p. Ioo à ceux des animaux des deux autres lots.
\end{abstract}

\section{INTRODUCTION}

L'ingestion de quantités importantes d'un aliment ayant une forte concentration énergétique conditionne le succès du sevrage précoce des agneaux et l'obtention de vitesses de croissance élevées pendant la phase d'engraissement. Les caractéristiques physico-chimiques des rations, leurs qualités organoleptiques et leur facilité de préhension sont des éléments importants qui déterminent le niveau d'ingestion de ces rations ou, selon le terme proposé par MCCLYMONT (1967) et repris par JOURNET et JARRIGE (I970), leur " ingestibilité ". 
L'ingestibilité des céréales, constituants essentiels de la ration d'engraissement des jeunes animaux, a fait l'objet de quelques études dans le cas de l'agneau. HANKE et Jordan (1963), Pinot (résultats non publiés), Davies (r968) ont présenté des conclusions partielles et parfois contradictoires sur ce point ; elles résultent en effet de méthodes et de conditions expérimentales très différentes.

Hanke et Jordan (I963), Ørskov et al. (I969), Vermorei, et al. (I970) ont comparé l'utilisation énergétique des diverses céréales pour l'engraissement de l'agneau. Le maïs possède la valeur énergétique nette la plus élevée, et les produits terminaux de sa digestion sont particulièrement favorables à la lipogenèse et surtout à la protéinogenèse (VERMOREL et al., I970). Le Blé, bien qu'ayant une valeur énergétique supérieure à celle de l'orge, a une valeur alimentaire plus faible que celle de l'orge ou du maïs à cause de son appétibilité généralement considérée comme faible, en particulier pour l'engraissement de jeunes bovins (OLTJEN et al., 1967).

$\mathrm{Au}$ cours d'une première étude nous avions comparé les valeurs énergétiques nettes de diverses céréales pour l'engraissement de l'agneau en offrant des quantités limitées d'aliment (VERMOREI, et al., I970). Nous avons poursuivi cette comparaison par la mesure de l'ingestibilité de rations riches en céréales. Pour cela nous avons offert à volonté à des agneaux, outre du foin normal, un aliment concentré contenant l'une des trois céréales étudiées : orge, blé ou maïs. La céréale représentait $70 \mathrm{p}$. 100 de l'aliment concentré et $55 \mathrm{p}$. Ioo de la ration totale.

\section{MATÉRIEL ET MÉTHODES}

Les agneaux, élevés en lots, recevaient un aliment concentré composé de tourteau d'arachide, de la céréale étudiée à laquelle était associé, dans un schéma expérimental de type blocs, 1'un des trois suppléments minéraux utilisés dans une étude simultanée de l'urolithiase chez l'agneau. Outre cet aliment concentré, présenté sous forme de granulés de $5 \mathrm{~mm}$ de diamètre, les agneaux disposaient de foin ncrmal, tous deux distribués à volonté. Nous ne rapporterons pas ici les effets

TABIEAU I

Composition des aliments utilisés au cours de l'essai

\begin{tabular}{|c|c|c|c|c|}
\hline & $\begin{array}{c}\text { Aliment } \\
\text { Orge }\end{array}$ & $\begin{array}{l}\text { Aliment } \\
\text { Blé }\end{array}$ & $\begin{array}{l}\text { Aliment } \\
\text { Maïs }\end{array}$ & Foin \\
\hline Orge................ & 73 & - & - & - \\
\hline Blé $\ldots \ldots \ldots \ldots \ldots \ldots$ & -- & 73 & - . & - \\
\hline Maïs $\ldots \ldots \ldots \ldots \ldots \ldots$ & - & - & 71 & - \\
\hline Tourteau d'arachide ..... & 20 & 20 & 22 & - \\
\hline Complément minéral.... & 3 & 3 & 3 & - \\
\hline $\begin{array}{l}\text { Paille d'orge ou supplé- } \\
\text { ment minéral } \ldots \ldots \ldots\end{array}$ & 4 & $t_{t}$ & 4 & - \\
\hline Matière sèche $\ldots \ldots \ldots$. & 89,6 & 89,0 & 90,2 & 90,7 \\
\hline Matières azotćes totales.. & 22,2 & 23,7 & 21,5 & 10,0 \\
\hline Cellulose brute $\quad \ldots \ldots \ldots$ & 4,9 & 1,5 & $\therefore, 3$ & 30,2 \\
\hline CUD de la matière sèche. & 81,8 & 86.6 & 83,8 & 50,3 \\
\hline
\end{tabular}


des compléments minéraux puisqu'ils n'ont pas présenté d'interaction avec la nature de la céréale. Un seul animal du lot recevant l'aliment maïs a été abattu, au poids vif de $30,5 \mathrm{~kg}$, après avoir présenté dos calculs urinaires.

Nous avons utilisé pour cette expérience 63 agneaux mâles $(45$ de race Limousine, I 8 croisés Limousin $\times$ Romanov) répartis en 9 lots de 7 animaux chacun. Ces agneaux élevés sous la mère ont été sevrés à 8 semaines; au début de l'expérience, âgés de 66 jours, ils pesaient $20 \mathrm{~kg}$ en moyenne.

Les aliments étaient distribués une fois par jour, le matin. Ils restaient en permanence à la disposition des animaux. Les refus, maintenus entre ro et 15 p. ıoo des quantités offertes, étaient recueillis et pesés chaque jour.

La teneur en matière sèche des rations a été déterminée une fois par semaine. Le tableau I présente les constituants et la composition des aliments ainsi que leur digestibilité mesurée in vitro par la méthode de Tilley et Terry (1963).

Les agneaux ont été pesés toutes les semaines et abattus dès que leur poids vif dépassait $35 \mathrm{~kg}$ ou, au plus tard, à l'âge de $\mathbf{3} 30$ jours pour ceux qui avaient eu la croissance la moins rapide. L'état d'engraissement des carcasses a été estimé par l'épaisseur du gras de couverture au niveau de la première vertèbre lombaire, et par la pesée des dépôts adipeux périrénaux. La qualité du gras de couverture a également été notée de I à 3 d'après son aspect, après 24 heures de ressuyage.

\section{RÉSULTATS}

\section{Quantités ingérées}

L'évolution des quantités de matière sèche ingérées sous forme d'aliment concentré et de foin est présentée par la figure I. Les agneaux recevant l'aliment maiss ont consommé davantage d'aliment concentré que ceux des autres lots : $\mathrm{P}<0,00 I$ entre le "mais " et le " blé ", $0,05<\mathrm{P}<0$, Io entre les aliments mais et orge (tabl. 2). L'évolution des quantités ingérées a été différente selon la nature de la céréale. Les consommations d'aliment orge ont augmenté régulièrement pendant toute la durée de l'expérience. Celles des aliments maïs ou blé se sont stabilisées lorsqueles agneaux ont dépassé le poids de $30 \mathrm{~kg}$.

Les consommations de foin sont restées relativement stables tout au long de l'essai. Les agneaux recevant l'aliment maïs ont consommé moins de foin que ceux des autres lots $(\mathrm{P}<0,00 \mathrm{I})$ tandis que ceux qui disposaient de l'aliment blé en ont ingéré les quantités les plus importantes $(\mathrm{P}<0,05$ entre les agneaux des lots "blé " et " orge ").

Sur l'ensemble de l'expérience, les agneaux recevant l'aliment maïs ont consommé davantage de matière sèche par jour que ceux des autres lots $(\mathrm{P}<0,05)$ En fin d'expérience toutefois les consommations journalières sont devenues assez comparables.

L'évolution des quantités totales de matière sèche ingérées par $\mathrm{kg}$ de poids métabolique est présentée dans la figure 2 . En début d'expérience les quantités de matière sèche ingérées par les agneaux recevant le maïs sont plus élevées que celles des animaux recevant l'orge ou le blé. Par la suite, l'évolution des quantités ingérées est la même quelle que soit la nature de la céréale. Entre 25 et $30 \mathrm{~kg}$ de poids vif on peut ainsi observer une légère diminution des quantités ingérées qui se sont ensuite stabilisées à un niveaı de $90 \mathrm{~g}$ de matière sèche par $\mathrm{kg}$ de poids métabolique par jour (tabl. 2). 


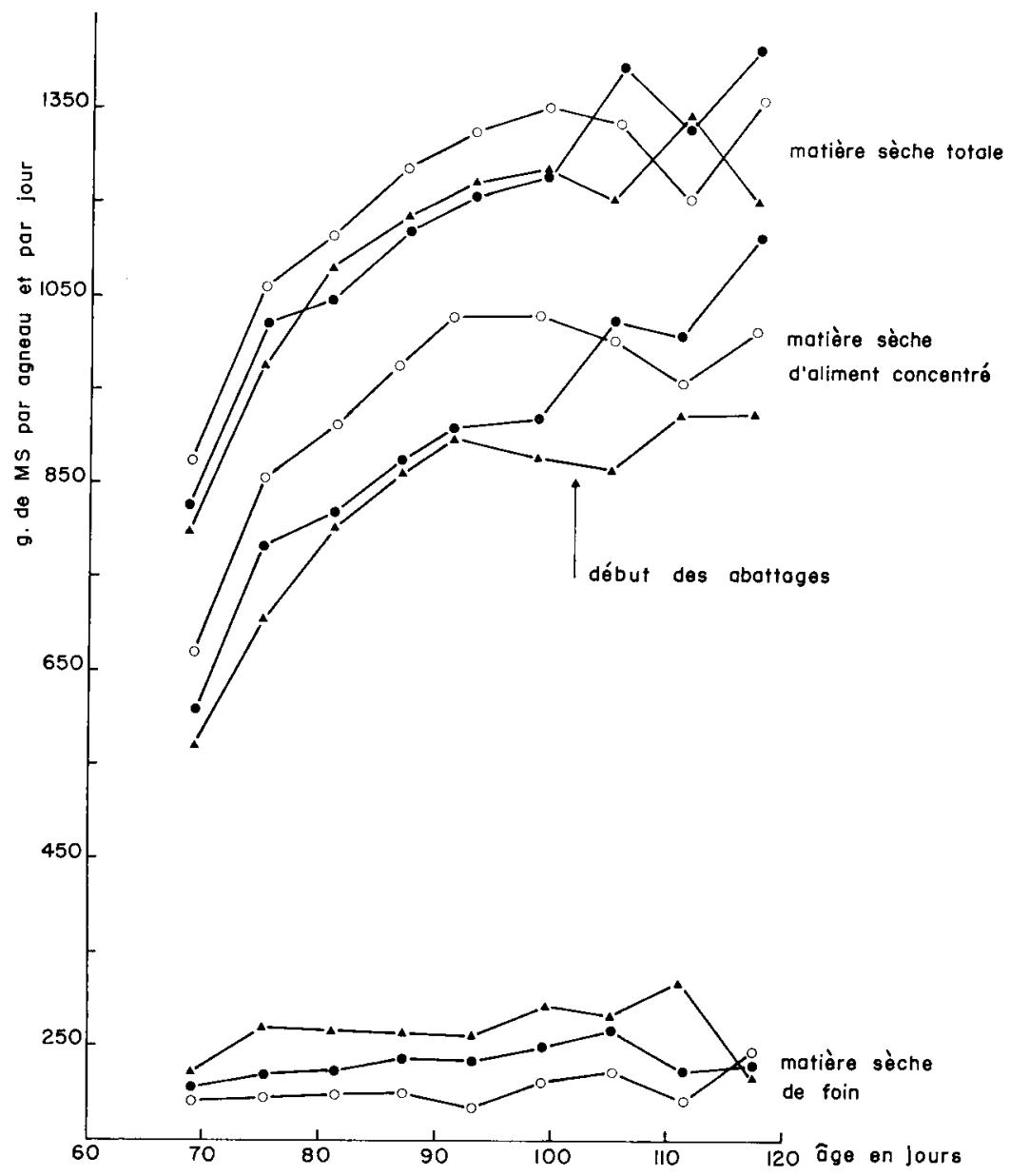

FIG. I. - Évolution des quantités de matière sèche consommées chaque jour dans la ration totale, sous forme d'aliment concentré ou de foin

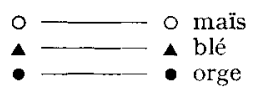

La consommation de foin diminue régulièrement tout au long de l'essai quel que soit le niveau initial. Par contre, la consommation d'aliment concentré par $\mathrm{kg}$ de $\mathrm{P}{ }^{0,75}$, augmente de $\mathbf{1} 5$ grammes au cours du premier mois pour se stabiliser à des valeurs légèrement inférieures aux maximums observés à l'âge de 90 jours. L'évolution est identique pour les 3 céréales. L'aliment blé est toujours moins consommé que l'aliment orge et la consommation d'aliment maïs est toujours la plus élevée.

La composition de la ration ingérée a donc varié d'un lot à l'autre et, pour un même lot, d'une période à 1'autre. La proportion d'aliment concentré a crû tout au long de l'essai dans la ration ingérée par les agneaux recevant les aliments blé ou orge. Par contre, après avoir atteint un maximum elle a diminué dans celle des agneaux recevant l'aliment maïs, dès que leur poids vif a dépassé $30 \mathrm{~kg}$. 


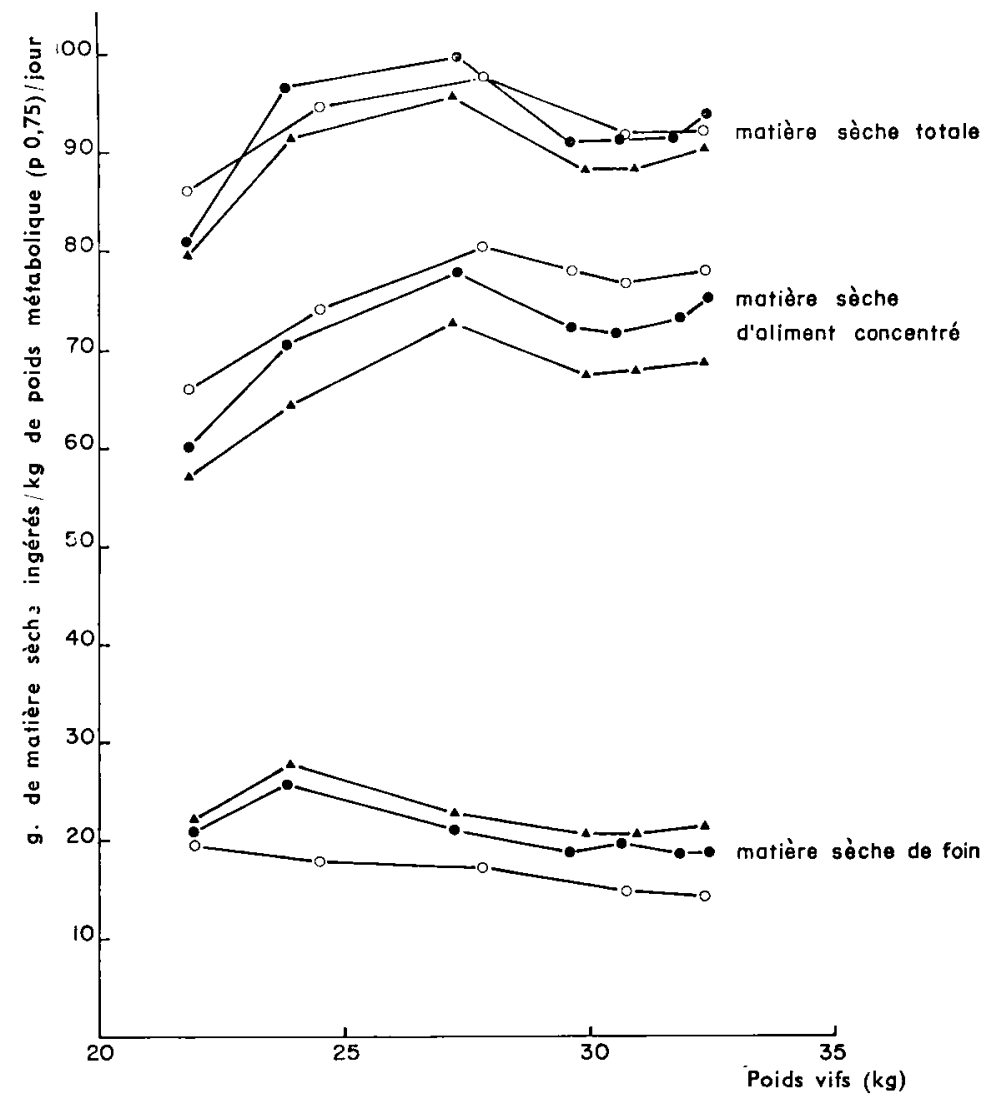

FIG. 2. - Évolution des quantités de matière sèche totale ingérées par jour et par $k g$ de poids métabolique $\left(\mathrm{P}^{0,75}\right)$

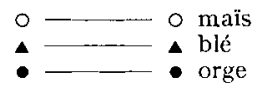

Pour l'ensemble de l'expérience, l'aliment concentré a représenté respectivement 82,78 et $75 \mathrm{p}$. Ioo de la ration pour les régimes maïs, orge et blé sur la base de la matière sèche ; la part de céréales de ces mêmes rations était de 58,57 et 55 p. Ioo (tabl. 2).

\section{Croissance et carcasses}

Les agneaux ont eu dans l'ensemble un gain de poids vif élevé, supérieur à $300 \mathrm{~g}$ par jour. Le régime à base de maïs a permis un gain quotidien moyen de $340 \mathrm{~g}$ au lieu de 306 et $302 \mathrm{~g} /$ jour pour les agneaux recevant respectivement les aliments blé et orge $(\mathrm{P}<0,05$ entre les lots disposant d'aliment orge et d'aliment mais). L'évolution du poids vif moyen des animaux est présentée sur la figure 3.

Les agneaux des 3 lots ont été abattus au même poids vif moyen de $35 \mathrm{~kg}$ à un âge moyen de II 2 jours pour ceux qui recevaient l'aliment mais, II 7 jours pour les autres (différence non significative) (tabl. 2). Ils ont produit les carcasses les plus lourdes $(\mathrm{P}<\mathrm{O}, \mathrm{Or})$, traduisant un meilleur rendement $(\mathrm{P}<0,00 \mathrm{r})$. 
TABIEAU 2

Déroulement et résultats de l'essai

\begin{tabular}{|c|c|c|c|}
\hline & Orge & Blé & Maïs \\
\hline \multicolumn{4}{|l|}{ Mise en lots } \\
\hline Age $(\mathrm{j}) \ldots \ldots \ldots \ldots \ldots$ & $\pm 6,5(1)$ & $\pm 1,1$ & $66,0 \pm 2,0$ \\
\hline Poids vif $(\mathrm{kg}) \quad \ldots \ldots \ldots \ldots \ldots$ & $20,3 \pm 2,9$ & $20,3 \pm 3,2$ & $20,1 \pm 2,8$ \\
\hline \multicolumn{4}{|l|}{ Composition de la ration ingérée } \\
\hline 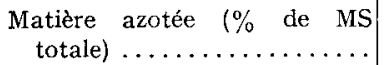 & 19,5 & 20,3 & 19,4 \\
\hline Cellulose brute ( $\%$ MS totale) & 10,4 & 10,9 & 8,9 \\
\hline \multicolumn{4}{|l|}{ Matière sèche ingéréelagneaulj } \\
\hline Aliment concentré (g de MS) & $845 *$ & $798 * *$ & 910 \\
\hline$(\%$ MS totale $)$ & 78 & 75 & 82 \\
\hline Foin ( $g$ de $M S) \quad \ldots \ldots \ldots \ldots$ & $234^{* *}$ & $267 * *$ & 204 \\
\hline$(\%$ de MS totale $) \ldots .$. & 22 & 25 & 18 \\
\hline Céréale ( $\%$ de MS totale) $\ldots$. & 57 & 55 & 58 \\
\hline \multicolumn{4}{|l|}{ Matière sèche ingêrée } \\
\hline Quantité totale & & & \\
\hline - $\mathrm{kg} / 100 \mathrm{~kg}$ de poids vif.... & 4,03 & 3,89 & 3,95 \\
\hline$-\mathrm{g} / \mathrm{kg} \mathrm{P^{0 } , 7 5} \ldots \ldots \ldots \ldots \ldots$ & 94 & 90 & 92 \\
\hline $\begin{array}{l}\text { Sous forme d'aliment concentré } \\
\qquad\left(\mathrm{g} / \mathrm{kg} \text { de } \mathrm{P}^{0},{ }^{75}\right) \ldots \ldots \ldots \ldots \ldots\end{array}$ & 73,3 & 67,5 & 75,5 \\
\hline \multicolumn{4}{|l|}{$\begin{array}{c}\text { Matière sèche ingérée par } k g \\
\text { de gain de poids vif }\end{array}$} \\
\hline Matière sèche totale $\ldots \ldots \ldots$ & 3,57 & 3,48 & 3,27 \\
\hline Matière sèche de céréale... & 2,05 & 1,95 & 1,91 \\
\hline Durée d'engraissement $(\mathrm{j})$. & $50,,^{\prime} \pm 11,1$ & $50,3 \pm 12,3$ & $46,3 \pm 11,6$ \\
\hline Gain de poids vif $(\mathrm{kg} / \mathrm{j}) \ldots \ldots$ & $302 \pm 58^{*}$ & $306 \pm 62$ & $340 \pm 54$ \\
\hline \multicolumn{4}{|l|}{ Abattage } \\
\hline Poids vif à jeun $(\mathrm{kg}) \ldots \ldots \ldots$ & $32,2 \pm 1,80$ & $32,5 \pm 2,07$ & $32,8 \pm 0,91$ \\
\hline Age $(j) \ldots \ldots \ldots \ldots \ldots$ & $117 \pm 11$ & $117 \pm 12$ & $112 \pm 12$ \\
\hline Poids de carcasse froide $(\mathrm{kg})$ & $14,96 \pm 1,27 * *$ & $14,59 \pm 1,12^{* *}$ & $15,74 \pm 0,64$ \\
\hline Rendement $\ldots \ldots \ldots \ldots \ldots$ & $46,50 \pm 2,32 * * *$ & $4 \mathbf{4}, 96 \pm 1,78 * * *$ & $47,95 \pm 1,65$ \\
\hline Épaisseur du gras dorsal (mm) & $2,73 \pm 0,73^{*}$ & $2,95 \pm 1,04^{*}$ & $3,55 \pm 1,21$ \\
\hline Gras périréılal $(\mathrm{g}) \ldots \ldots \ldots \ldots$ & $194 \pm 65^{* *}$ & $162 \pm 52 * *$ & $24 \pm 79$ \\
\hline Gain de poids de carcasse $(\mathrm{g} / \mathrm{j})$ & $133^{*}$ & $1 \geq 6^{*}$ & 162 \\
\hline 10 & & & \\
\hline
\end{tabular}

(1) Écart type de la moyenne. Signification des différences observées : tous les écarts sont rapportés au maïs : * $\mathrm{P}<0,05 \quad * * \mathrm{P}<0,01 \quad * * * \mathrm{P}<0,001$ 


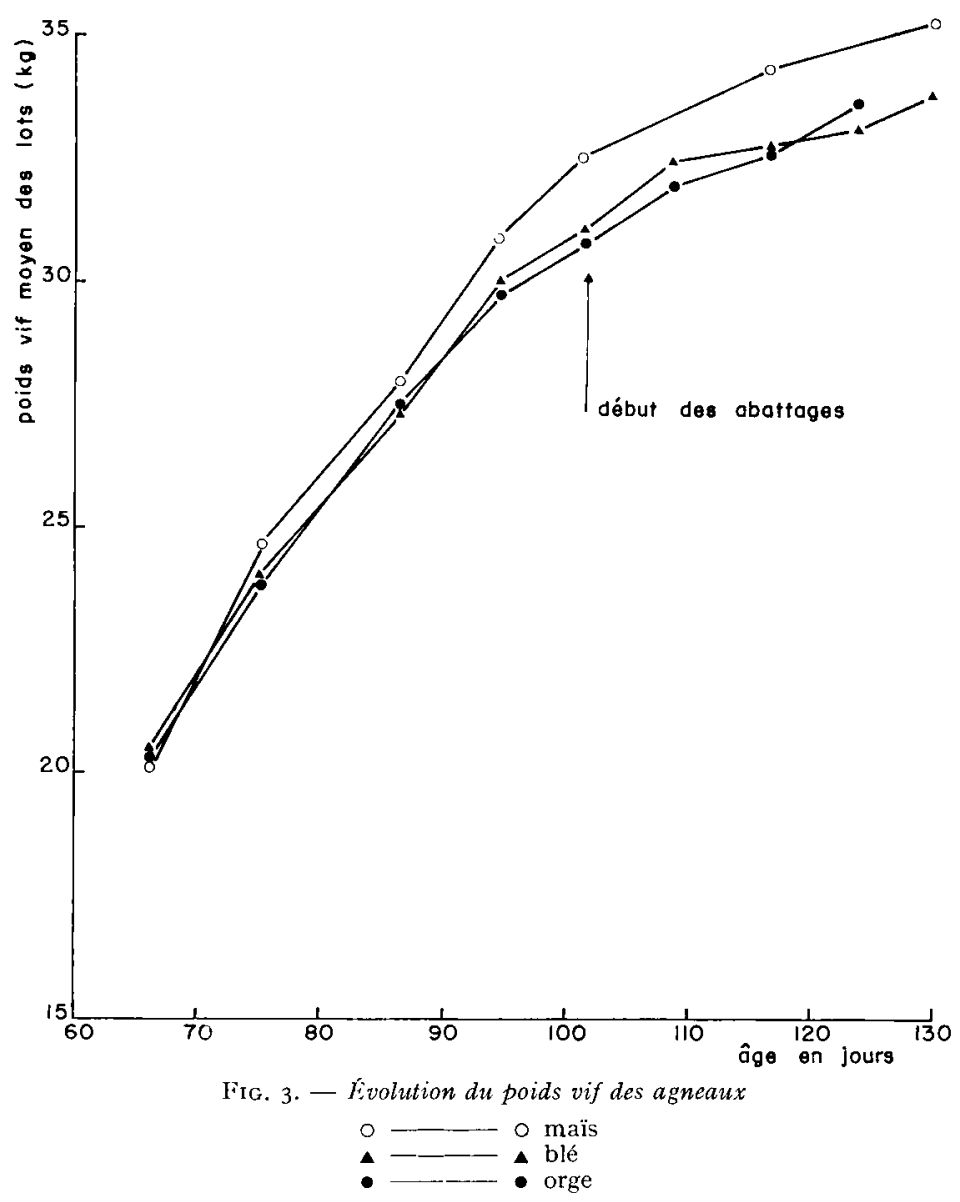

Nous avons estimé le poids de carcasse des animaux au début de l'expérience d'après leur poids vif et le rendement moyen de I9 agneaux de même race, d'âge et de poids identiques, abattus précédemment (rendement de 40,I3 $\mp 33,7$ p. 100). A partir de ces données nous avons pu déterminer que les agneaux ayant reçu l'aliment maïs ont gagné $162 \mathrm{~g}$ de carcasse par jour contre respectivement I33 et $\mathrm{I} 26 \mathrm{~g}$ pour ceux des lots " orge " et "blé » $(\mathrm{P}<0,05)$.

L'estimation de l'état d'engraissement, par la mesure de l'épaisseur du gras dorsal, n'a fait apparaître aucune différence entre les agneaux recevant l'orge ou le blé. Par contre, les agneaux consommant l'aliment maïs ont présenté un dépôt adipeux de couverture plus épais $(\mathrm{P}<0,05)$ et un gras périrénal plus lourd en moyenne $(\mathrm{P}<0,00 \mathrm{I})$ que ceux des autres lots.

Les agneaux ont ingéré respectivement $3,57,3,48$ et $3,27 \mathrm{~kg}$ de matière sèche soit 2,05 , I,95 et I,9I $\mathrm{kg}$ de matière sèche sous forme de céréale par $\mathrm{kg}$ de gain de poids vif pour les lots disposant d'aliment orge, blé ou mais. Rapportées au $\mathrm{kg}$ de gain de carcasse froide, les quantités ingérées sont de 4,40,4,34 et 3,98 kg sous forme de matière sèche de céréales et de $8,04,8,53$ et $6,88 \mathrm{~kg}$ de matière sèche totale. Dans 
ce dernier cas, il convient toutefois de souligner que les rations ingérées contenaient des proportions variables de fourrage grossier.

\section{DISCUSSION}

Les agneaux, disposant d'aliment concentré et de foin de pré de qualité médiocre offerts tous deux ad libitum, ont choisi des rations composées de 23 p. Ioo environ de foin. Cette valeur correspond à celle qu'ont observée PiNot et JARRIGE (Ig68) dans un essai au cours duquel ils ont offert à des agneaux Préalpes, entre $2 \mathrm{I}$ et $35 \mathrm{~kg}$ de poids vif, du foin de luzerne de bonne qualité sous forme normale et un aliment concentré en granulés. La proportion de 75 p. Ioo d'aliment concentré, 25 p. Ioo de foin long, semble caractéristique des régimes d'engraissement d'agneaux de races rustiques et ceci malgré des différences importantes quant à la nature et à la qualité du foin offert.

Ce rapport moyen varie cependant selon le poids des animaux et la nature de la céréale. Il augmente au fur et à mesure que l'animal est plus lourd passant respectivement de 69 à 76 p. I00, de 73 à 80 et de 8 I à 84 p. Ioo pour les agneaux recevant les aliments blé, orge et mais. Cette évolution, due à une augmentation de la quantité d'aliment concentré ingéré au cours de l'engraissement tandis que la consommation de foin reste stable quel que soit le poids de l'agneau, a été également observée par PinO'T et JARRIGE (I968) avec un foin de luzerne de bonne qualité. Dans leur cas la proportion d'aliment concentré est passée de 70 à 78 p. Ioo de la ration. L'aliment concentré à base de maīs a eu tout au long de l'essai une ingestibilité élevée, supérieure à celle des deux autres. Ceci confirme des résultats non publiés de Pinot, obtenus pourtant selon une méthode différente (MATHIEU et WÉGaT-Litré, I96I). Par contre, l'aliment blé, bien qu'ayant une digestibilité de la matière sèche plus élevée, a une ingestibilité réduite déjà signalée par ailleurs chez les ruminants (THIVEND, I969) et chez le Porc (LAWRENCE, I967; HenRy, I968). Selon ces derniers auteurs, l'état granulométrique et la structure physico-chimique de cette céréale joueraient un rôle important dans la modification de l'ingestibilité des rations qui en contiennent de fortes proportions, du moins chez le Porc. D'autre part, nous n'avons noté aucun accident digestif comparable à ceux que rapportent OLTJEN et al. (I966) avec des rations ne contenant pas de foin normal, et les croissances obtenues avec cette céréale ont été très satisfaisantes $(302 \mathrm{~g} / \mathrm{j})$.

L'ingestibilité de l'aliment orge est intermédiaire, elle augmente cependant au cours de l'expérience pour atteindre des valeurs voisines de celles de 1'aliment maîs. Les faibles valeurs observées sur de jeunes agneaux ne semblent donc pas dues à une appétibilité réduite de l'orge mais à sa teneur en cellulose brute et à la digestibilité de celle-ci.

Au-delà du poids vif de 27 à $30 \mathrm{~kg}$, les agneaux ont limité leur consommation de matière sèche tout particulièrement sous forme d'aliment concentré. La composition de la ration ingérée entre 27 et $32 \mathrm{~kg}$ de poids vif fait apparaître des écarts importants entre les niveaux d'énergie digestible ingérée, malgré une ingestion plus élevée de foin par les agneaux recevant les aliments blé ou orge (fig. 2). 
En effet, si l'on attribue aux divers constituants des rations les valeurs énergétiques qui sont couramment admises (KELLNER et BECKER, I966; DemarquiLLy et Werss, I970), on constate que les agneaux recevant les aliments orge ou blé ont ingéré des quantités journalières de matière sèche et d'énergie sensiblement égales. Le régime à base de maïs a apporté un supplément énergétique d'environ $\mathrm{I}_{5} \mathrm{p}$. Ioo qui est dû à sa valeur énergétique et à son ingestibilité supérieures. Ceci s'est traduit par une amélioration équivalente du gain de poids vif. Par contre, compte tenu des rendements supérieurs des agneaux recevant le maïs, observation qui est conforme à celles de Preston et al. (I963) et de Vermorei, et al. (I970), le gain de carcasse journalier a été supérieur de 28 p. Ioo à celui observé pour les aliments orge et blé. Outre la valeur énergétique supérieure du mais, les produits terminaux de sa digestion ont donc été mieux utilisés que ceux des autres céréales pour l'engraissement de l'agneau. Ceci confirme les travaux de ALLEN (I969) et VERMOREL et al. (I970), mais n'est pas en accord avec les données classiques sur la valeur énergétique des différentes céréales (KELLNER et BECKER, I966).

L'appétibilité du maîs a entraîné chez les agneaux une consommation d'énergie supérieure à celle des animaux des lots orge et blé. Si la quantité de matière sèche ingérée sous forme d'aliment concentré par $\mathrm{kg}$ de poids métabolique s'est bien stabilisée lorsque le poids vif a dépassé $27 \mathrm{~kg}$, le niveau d'ingestion est resté, par contre, plus élevé pour le maïs. La satisfaction des besoins énergétiques ne semble pas être dans ce cas le seul mécanisme régulateur. Au même titre que la concentration énergétique de la ration (ANDREws et al., I969; OWEN et al., I969), des facteurs tels que l'appétibilité ou la structure physico-chimique des aliments petivent moduler la régulation de l'appétit même si le volume du rumen n'est plus le facteur limitant les quantités ingérées.

La qualité de la carcasse et du gras de couverture n'a pas été affectée par la nature de la céréale. L'état d'engraissement supérieur des agneaux élevés au maïs est certainement dî, au moins en partie, à leur poids de carcasse plus élevé lors de l'abattage ; cet état d'engraissement n'est d'ailleurs pas excessif et ne pose pas de problèmes particuliers de commercialisation. De plus, contrairement à ce que nous avions observé par ailleurs, les dépôts adipeux n'ont présenté aucune coloration jaune.

Si le maïs a permis d'obtenir des résultats particulièrement intéressants, l'orge et le blé peuvent également être utilisés dans des rations d'engraissement pour agneaux. Nous n'avons observé qu'un cas de lithiase et aucun des accidents digestifs ou hépatiques signalés chez les animaux recevant des rations contenant une forte proportion de blé (OLTJEN et al., I966) ou d'orge (OWEN et al., I967) ; mais, contrairement à celles dont disposaient les agneaux que nous avons utilisés, ces rations ne contenaient pas de foin normal.

Sous la forme de présentation que nous avons adoptée, le maïs est la céréale la plus favorable à l'engraissement de l'agneau. I1 présente en effet une ingestibilité plus élevée que celle des autres céréales étudiées en particulier chez l'animal de moins de Ioo jours ou de moins de $30 \mathrm{~kg}$ de poids vif. En fin d'engraissement, surtout si l'on cherche à obtenir des carcasses lourdes $(20 \mathrm{~kg})$, l'orge ou le blé pourront lui être substitués sans entraîner de réduction trop sensible de consommation ou de vitesse de croissance. 


\section{SUMMARY}

\section{COMPARATIVE UTILIZATION OF WHEAT, BARLEY}

\section{AND MAIZE IN THE FATTENING LAMB DIETS}

The food intake levels and utilization of rich cereal diets (barley, wheat or maize) were compared with 63 males Limousin lambs, reared in groups, weighing $20 \mathrm{~kg}$ at the beginning of the experiment and slaughtered at a mean live weight of $32 \mathrm{~kg}$. The lambs were fed concentrate diets (73 p. Ioo cereals) and long hay, both offered ad libitum (table I).

I. The lambs ingested similar amounts of total dry matter per $\mathrm{kg}$ metabolic weight $\mathrm{P}^{\mathbf{0 . 7 5}}$; but they constituted diets of variable composition according to the nature of the cereal (fig. I, table 2 ).

2. The amounts of dry matter of the concentrate ingested per $\mathrm{kg} \mathrm{P}^{\mathbf{0 . 7 5}}$ was always higher in the lambs receiving maize than in those receiving barley or wheat. This last cereal was less consumed than the others (fig. 2).

3. The amounts of hay ingested per $\mathrm{kg} \mathrm{P}^{0.75}$ decreased during the trial and constituted on average, $18 \mathrm{p}$. Ioo of the dry matter intake per day in the lambs receiving maize, instead of 22 and $25 \mathrm{p}$. Ioo in those receiving barley and wheat respectively.

4. During the first month, between 20 and $27 \mathrm{~kg}$ live weight, we observed an increase of the dry matter intake per $\mathrm{kg} \mathrm{P}^{0.75}$ followed by a decrease and a stabilization of the intake levels at about 90 g per $\mathrm{kg} \mathbf{P}^{\mathbf{0 . 7 5}}$ and per day.

5. The lambs consumming the " maize " concentrate, ingested 15 p. Ioo more energy (calculated in FU) per day than the animals fed "barley " or "wheat " concentrates. This resulted in a similar difference in the live weight gains (340 g/day versus 302 and $306 \mathrm{~g} / \mathrm{day}$, fig. 3 ). On the other hand, the maize fed animals showed daily carcass gains $28 \mathrm{p}$. roo higher. This clearly shows that the terminal products, arising from the digestion of maize, are well utilized by the fattening lamb.

6. The factors, responsible for the differences between the three cereals, which, in the case of maize, could induce a higher consumption of digestible energy than that necessary to meet the requirements for energy, are discussed.

\section{RÉFÉRENCES BIBLIOGRAPIIQUES}

Allen D. M., r969. The performance of lambs fed all concentrate diets varying in energy density and crude protein content. in Meat Prod. from entire male animal. D. N. Rhodes éd., p. I03-Io8.

Andrews R. P., KAY M., ORskov E. R., I969. The effect of different dietary energy concentrations on the voluntary intake and growth of intensively fed lambs. Anim. Prod., 11, I.73-I85.

Davies P. J, 1968. The effect of cereal and protein source on the energy intake and nitrogen balance of fattening lamb given all concentrate diets. Anim. Prod. 10, 31 I-318.

Demarquilly C., Weiss Ph,, r970. Tableaux de la valeur alimentaire des fourrages. Étude S. E. I., $n^{\circ} 42$.

Hanke H. F., Jordan R. M., I963. Comparison of lambs fed shelled corn and whole pelleted barley of different bushel weights. J. Anim. Sci, 22, 1097-1099.

HENRY Y., I968. Utilisation comparée des céréales comme seuls aliments du Porc pendant la période de finition. Ann. Zootech., 17, I83-197.

Journet M., JaRrige R., I970. I’acteurs physiologiques de la quantité d'aliments ingérée par les ruminants, $21^{\mathrm{e}}$ Réunion annuelle de la Fédération Européenne de Zootechnie. Budapest, 24-28 août I970.

Kellner O.. Becker M., I966. Grundzïge der Fitterungslehre (14 ${ }^{\mathrm{e}}$ ed.) Verlag Paul Parey. Hamburg, Berlin.

LAwrence T. L. J., I967. High level cereal diets for the growing finishing pig. The effect of cereal preparation and water level on the performance of pigs fed diets containing high levels of wheat. J.agric. Sci., 68, 269-274. 
Mathiev C.-M., Erna WÉGat-Litré, I960. Les préférences alimentaires du veau. I. Appétibilité comparée des céréales. Ann. Zootech., 9, $261-270$.

McClymont G. L., 1967. Selectivity and intake in the grazing ruminant. in Handbook of physiology, Section 6. Alimentary Canal. Vol. 1, I29-137.

Pinot R., Jarrige R., I968. Utilisation des aliments broyés et agglomérés par l'agneau à l'engrais. II. Comparaison du foin de luzerne condensé au foin de luzerne normal en présence d'un aliment concentré offert ad libitum. Ann. Zootech., 17, 5-22.

Oltjen R. R., Putnam P. A., Williams E. P., Davis R. F., rg66. Wheat versus corn in all concentrate cattle rations. J. Anim., Sci, 25, I000-I004.

$\emptyset_{\text {RSKOV }}$ E. R., FRAser C., KAY R. N. B., I969. Dietary factors influencing the digestion of starch in the rumen and small and large intestive of early weaned lambs. Br. J. Nutr., 23, 2I7-226.

Owen J. B., Davies D. A. R., Mrller E. L., Ridgman W. J., I967. The intensive rearing of lambs. II. Voluntary food intake and performance on diets of varying oat husks and beef tallow content. Anim. Prod., 9, 509-5 I9.

Owen J. B., Davies D. A. R., Ridgman W. J., Ig69. The control of voluntary food intake in ruminants. Anim. Prod., 11, 5II-520.

Preston T. R., Aitien J. N., Whitelaw F. G., Mac Dearmid A., Euphemia Philip B., Mac Leod N. A., I963. Intensive beef production. III. Performance of Friesian steers given low fibre diets. Anim. Prod. 5, $245 \cdot 249$.

Thivend P., I969. Le blé dans l'alimentation du Ruminant. Bull. Tech. Inf., 244, 935-940.

Triney J. A. M., Terry R. A., I963. A two stage technique for the in vitro digestion of forage crops. J. Brit. Grass. Soc., 18, IO4-III.

Vermorel M., Thivend P., Theriez M., i97o. Utilisation énergétique de l'orge, du blé, du maîs et du sorgho par l'agneau en croissance. Ann. Zootech., 19, 46I-464. 